\title{
Relationship Between Periodontitis and Rheumatoid Arthritis and the Effect of Non-Surgical Periodontal Treatment
}

\author{
Márcia de Noronha PINHO ${ }^{1}$ \\ Renê Donizeti Ribeiro OLIVEIRA ${ }^{2}$ \\ Arthur Belém NOVAES JR. ${ }^{3}$ \\ Júlio César VOLTARELLI ${ }^{2}$
}

\author{
${ }^{1}$ Dental School of Campinas, Pontifical Catholic University, Campinas, SP, Brazil \\ ${ }^{2}$ Ribeirão Preto Medical School, University of São Paulo, Ribeirão Preto, SP, Brazil \\ ${ }^{3}$ Ribeirão Preto Dental School, University of São Paulo, Ribeirão Preto, SP, Brazil
}

\begin{abstract}
This study analyzed the association of periodontal disease (PD) and rheumatoid arthritis (RA). Seventy-five 35-60-year-old patients were assigned to 5 groups according to the presence (+) or not (-) of PD and RA and the treatment received (TR+) or not (TR-) for PD. Group 3 uses total prosthesis (TP). Clinical and laboratory evaluations were performed at baseline, 3 and 6 months of follow-up by probing pocket depth, bleeding on probing and plaque index for PD, HAQ, DAS28, SF-36 and laboratory: AAG, ESR, CRP for RA. Statistically significant differences for PD after $3(\mathrm{p}=0.0055)$ and after 6 months $(\mathrm{p}=0.0066)$ were obtained in Group $1(\mathrm{RA}+\mathrm{PD}+\mathrm{TR}+)$ and 2(RA+PD+TR-); significant reduction in the $\%$ of BOP after 6 months $(\mathrm{p}=0.0128)$ and significant reduction in the $\%$ of $\mathrm{Pl}$ after $3(\mathrm{p}=0.0128)$ and 6 months $(\mathrm{p}=0.0002)$ in Group 1. Statistically significant differences between Groups 1 and 3 (RA+TP) for DAS28 at baseline and after 3 months were observed, but not after 6 months. No other parameters for RA were significantly affected. The relationship between RA and PD disease activities is not clear, but the importance of periodontal treatment in the control of inflammation to avoid tooth extraction is evident.
\end{abstract}

Key Words: rheumatoid arthritis, periodontal disease, periodontal medicine, non-surgical periodontal treatment, acute phase proteins, questionnaires of disease activity.

\section{INTRODUCTION}

Periodontal disease (PD) shares several clinical and pathogenic characteristics with chronic arthritis. Periodontal diseases are not only a threat to dentition, but may also be a threat to general health. There are reports suggesting increased prevalence of diabetes, atherosclerosis, myocardial infarction, stroke, and rheumatoid arthritis (RA) in patients with periodontal disease $(1,2)$. All forms of inflammatory periodontal diseases are associated with chronic inflammation (accumulation of $\mathrm{B}$ and $\mathrm{T}$ lymphocytes as well as of monocytes and neutrophils), resulting in the destruction of periodontal ligament and bone (3). The current paradigm for RA includes an initiating event leading to significant synovial inflammation and tissue destruction. As for periodontitis, there is an accumulation of inflammatory cells in the gingiva (T and B lymphocytes, neutrophils, and monocytes), tissue edema, endothelial cell proliferation, and matrix degradation (4).

The similarities between RA and PD have prompted several studies on the periodontal status of patients with RA although the relationship between RA and PD that emerged from those studies are controversial (5-8). In light of these reports, there is a need for further investigation to determine whether the severity of RA and PD is interrelated. A possible correlation between periodontal disease and rheumatoid arthritis and an improvement in both conditions following non-surgical periodontal treatment would bring benefits to the population with both diseases. Thus, the aim of this study is to analyze, clinically and systemically, the association 
between PD and RA, and if non-surgical periodontal treatment has any effect on the control of RA.

\section{MATERIAL AND METHODS}

This clinical study was carried out as a joint collaboration between the Division of Clinical Immunology of the Medical School and the Department of Periodontology of Ribeirão Preto Dental School, University of São Paulo, SP, Brazil.

The study population consisted of a sample of 75 patients, ranging from 35-60 years of age, which were assigned into 5 Groups of 15 patients each, according to the presence of the systemic diseases (RA) and/or periodontal diseases (PD) and to the treatment or not of the PD (TR). The Groups were: Group 1: patients with diagnosis of RA and PD who received non-surgical periodontal treatment (NSPT) during the study RA+PD+TR+ (random allocation with Group 2); Group 2: patients with diagnosis of RA and PD who were monitored during the study and did not receive periodontal treatment RA+PD+TR-; Group 3: patients with diagnosis of RA and using total prosthesis RA+TP; Group 4: patients with diagnosis of PD without RA analyzed before and after non-surgical periodontal treatment RA-PD+TR +; Group 5: systemic and periodontally healthy patients RA-PD-.

The protocol was approved by the Ethics Committee on Human Research of the University Hospital of Ribeirão Preto Medical School (Protocol \#1538.0.004.000-05) and all patients gave written informed consent for the treatment. The arthritis patients were selected according to the criteria of the American College of Rheumatology (9) for the diagnosis of RA and were treated at the University Hospital.

The period from diagnosis of RA until the enrollment in the study varied from 6 months to 10 years. The medication used by the patients was maintained, to avoid study interferences. The patients belonged to the same low income class.

The patients with periodontal disease had 2 or more teeth with attachment loss $\geq 6 \mathrm{~mm}$ and one or more teeth with probing pocket depth $\geq 5 \mathrm{~mm}$, according to the criteria of Machtei et al. (10). Considering the dentate groups the number of teeth was about 20 in each patient.

Exclusion criteria were smoking, pregnant or nursing women and patients with any systemic disease or who had used antibiotics within the previous 3 months.
At baseline, medical and dental histories were recorded and various parameters were measured. Periodontal clinical parameters involved were: probing pocket depth (PPD) measured at 6 sites/teeth using an electronic probe (Florida Probe Corporation, Gainesville, FL, USA), the percentage of sites presenting bleeding on probing (BOP) and the percentage of teeth surfaces which revealed the presence of dental plaque (PL).

The activity of the RA was measured by laboratory tests and clinical evaluation (questionnaires of disease activity and physical examination).

\section{Laboratory Methods}

Rheumatoid factor (RF): IgM rheumatoid factor was determined by nephelometry and used only as inclusion criteria. These values were extracted from the patients records.

Acute-phase reaction tests: *alpha - 1 acid glycoprotein (AAG): the concentration of AAG was determined by kinetic nephelometry. The normal value was less than $120 \mathrm{mg} \%$; *The erythrocyte sedimentation rate (ESR): it was measured by the Wintrobe method, normal values are less than $10 \mathrm{~mm} / 1^{\text {st }} \mathrm{h}$ for men and less than $20 \mathrm{~mm} / 1^{\text {st }} \mathrm{h}$ for women; ${ }^{*} \mathrm{C}$-reactive protein $(\mathrm{CRP})$ : the concentration of CRP was determined by kinetic nephelometry. The normal value is less than $0.5 \mathrm{mg} / \mathrm{dL}$.

\section{Questionnaires of Disease Activity}

The Stanford Health Assessment: the HAQ has been established as a valuable, effective, and sensitive tool for measurement of health status.

Disease Activity Score (DAS28): DAS stands for "Disease Activity Score", and measures the activity of rheumatoid arthritis. The following parameters are included in the calculation: number of joints that are tender to the touch (TEN), number of swollen joints (SW), erythrocyte sedimentation rate (ESR) and patient assessment of disease activity (VAS; mm).

Short-Form Health Survey (SF 36): SF-36 is a multipurpose questionnaire, with only 36 questions. It yields an 8 -scale profile (A-H) of functional health and well-being scores as well as a psychometrically-based physical and mental health summary and a preference-based health utility index. It is a generic measure, as opposed to one that targets a specific age, disease, or treatment group. An increased value of this data reflects patients' improvement. 
All of these examinations and tests were made at baseline, 3 and 6 months after non-surgical periodontal treatment in Group $1(\mathrm{RA}+\mathrm{PD}+\mathrm{TR}+)$ and after start monitoring of Group 2 (RA+DP+TR-). The patients from Group 2, who did not receive periodontal treatment during the study were treated after the end of the study. The questionnaires of disease activity showed the functional capacity of the patients, before and after periodontal treatment. In Group 4 (RA-PD+TR+) the same treatment was applied, without specific examinations for RA, only laboratory tests for acute-phase reaction (AAG, ESR, CRP). The patients were monitored monthly. In Group 3 (RA+TP), patients were examined just once, and all 15 patients had their teeth extracted because of severe periodontal disease. Group 5 (RA-PD-) was examined periodontally and systemically (acute-phase reaction) once. These 2 Groups were used as control groups of the study. The non-surgical periodontal treatment consisted of full-mouth conventional scaling and root planning (SRP).

The clinical and laboratory parameters collected received statistical analyses at baseline, 3 and 6 months post-treatment. The Mann-Whitney test was performed to compare the differences between Groups, and the Friedman Anova and Wilcoxon tests were performed to compare the differences between the examination periods (baseline, 3 and 6 months) in each Group. The Spearman correlation test was used to analyze associations between variables. All statistical analysis considered the significance level (p) of 5\%.

\section{RESULTS}

The present study did not have any sample loss; all the selected patients placed in their respective Groups finished the period of the study. In relation to the demographic data, the age average of the groups was 50 years old, and $60 \%$ of the patients were female, which reflected the RA incidence 3:1 (9).

\section{Intra-Group Analysis}

The PPD of group 1 (RA+PD+TR+) was statistically reduced for the entire 6 months post-treatment period ( $p<0.0043)$, as well as the $\%$ of PL $(p<0.0016)$. There was a reduction in the $\%$ of BOP, but it was not statistically significant $(p<0.0726)$. For the acute phase laboratory data, AAG, ESR and CRP there was no sta- tistical differences after 6 months (0-3, 0-6 and 3-6).

The Group 2 (RA+PD+TR-) did not show statistically significant reductions for the variables PPD ( $<<0.3678$ ), $\%$ of PL and $\%$ of BOP. Systemically, only ESR was statistically significant for the 6 months evaluation $(0-6)(\mathrm{p}=0.0007)$.

For Group 4 (RA-PD+TR+) all the variables were significantly reduced: PPD $(p<0.0001), \%$ of PL $(p<0.0000)$ and $\%$ of BOP $(p<0.0000)$. (Tables $1,2,3)$. Systemically, AAG $(p<0.0001), \operatorname{PCR}(p<0.0000)$, VHS $(p<0.0000)$ all had statistically significant reductions. These differences occurred in the examination periods of 0-3, 0-6, 3-6 months (Tables 6-8).

\section{Inter-Group Analysis}

The differences between Groups 1 and 4 were statistically significant for $\%$ of PL $(p=0.0152)$ for the evaluation between 3-6 months. Although the improvements were greater for Group 1, for PPD and \% of BOP, the differences were not statistically significant (Table 4). The differences for the systemic parameters AAG, ESR and CRP were statistically significant between Groups 1 and 4, AAG for the examination periods for $0-3$ and 0-6 months, CRP and ESR in the evaluation periods of 0-6, 3-6 months (Table 9).

The differences between Groups 1 and 2 were statistically significant for PPD after $3(\mathrm{~T} 1-\mathrm{T} 0)(\mathrm{p}=0.0055)$ and after 6 months (T2-T0) $(\mathrm{p}=0.0066)$; for the $\%$ of BOP it was significant after 6 months $(\mathrm{T} 2-\mathrm{T} 0)(\mathrm{p}=0.0128)$ and for the $\%$ of PL it was significant after 3 (T1-T0) $(p=0.0128)$ and 6 months $(T 2-T 0)(p=0.0002)$ in favor of Group 1, the group that received the non-surgical periodontal treatment (Table 5).

Comparing the initial PPD average from Group 4 to punctual data collected from control Group 5 (RA-PD-) there were no statistically significant differences. $(\mathrm{p}=0.6936)$. The other data showed statistically significant differences: $\%$ of BOP $(p=0.0000), \%$ of PL $(\mathrm{p}=0.0084), \mathrm{AAG}(\mathrm{p}=0.000), \mathrm{CRP}(\mathrm{p}=0.0020)$ and $\mathrm{ESR}$ $(\mathrm{p}=0.0001)$. After 3 months there were no differences for the $\%$ of PL $(p=0.0712)$ and PPD ( $p=0.4186)$, although for the other variables there were differences: AAG $(p=0.0000), \operatorname{CRP}(p=0.0171), E S R(p=0.0007)$ and $\%$ of BOP $(\mathrm{p}=0.000)$. After 6 months the PPD reduction for Group 4 was bigger and statistically significant when compared to the other groups $(p=0.0005)$, the $\mathrm{CRP}(\mathrm{p}=0.9670)$ and ESR $(\mathrm{p}=0.0591)$ values were no 
longer statistically different at this time. The differences in AAG ( $\mathrm{p}=0.0004)$ continued and there were statistical differences in: $\%$ of BOP $(\mathrm{p}=0.0017)$ and $\%$ of PL $(p=0.0001)$ (Table 10).

Comparing Group 1 (RA+PD+TR+) to Group 5 (RA-PD-) the variables AAG, $\%$ of BOP were statistically different for all 3 evaluation periods (baseline, 3 and 6 months), being bigger for Group 5. The PPD was different at baseline $(\mathrm{p}=0.0001)$ and after 3 months $(\mathrm{p}=0.0095), \%$ of PL only at baseline ( $\mathrm{p}=0.0004)$. CRP and ESR did not present statistical differences in any of the evaluations (Table 11). Comparing Group 1 (RA+PD+TR+) to Group $3(\mathrm{RA}+\mathrm{TP})$ there were statistically significant differences in DAS28 at baseline and after 3 months, bigger for Group 3 , but not after 6 months (Table 12).

In Group 4 correlations were found at the 0-3 months examinations when comparing $\%$ of BOP and AAG $(r=0.50 ; p<0.0281)$, when comparing PPD in the 0-3 months to AAG in the 3-6 months examinations $(r=0.80 ; p<0.0000)$ and when comparing $\%$ of PL in

Table 1. Mean probing pocket depth (mm) of 45 patients: 15 from Group 1 (RA+PD+TR+), 15 from Group 2 (RA+PD+TR-) and 15 from Group 4 (RA-PD+TR+).

\begin{tabular}{lccccccc}
\hline Patients & $\begin{array}{c}\mathrm{T} 0 \\
\text { Initial }\end{array}$ & $\begin{array}{c}\mathrm{T} 1 \\
\text { 3 months }\end{array}$ & $\begin{array}{c}\mathrm{T} 2 \\
6 \text { months }\end{array}$ & Friedman & $\begin{array}{c}\text { T1-T0 } \\
\text { Wilcoxon }\end{array}$ & $\begin{array}{c}\text { T2-T1 } \\
\text { Wilcoxon }\end{array}$ & $\begin{array}{c}\text { T2-T0 } \\
\text { Wilcoxon }\end{array}$ \\
\hline Group 1 & $3.24(0.88)$ & $2.82(0.77)$ & $2.71(0.76)$ & $\mathrm{p}<0.0043$ & $\mathrm{p}=0.0096$ & $\mathrm{p}=0.1579$ & $\mathrm{p}=0.0030$ \\
Group 2 & $3.12(0.47)$ & $3.12(0.47)$ & $3.13(0.45)$ & $\mathrm{p}<0.3678$ & $\mathrm{p}=0.8886$ & $\mathrm{p}=0.8785$ & $\mathrm{p}=0.2135$ \\
Group 4 & $2.46(0.47)$ & $2.11(0.51)$ & $1.87(0.39)$ & $\mathrm{p}<0.0001$ & $\mathrm{p}=0.0018$ & $\mathrm{p}=0.0007$ & $\mathrm{p}=0.0309$ \\
\hline
\end{tabular}

Mean (standard deviation). Kruskal-Wallis ANOVA (testing overall difference among the three groups in the baseline - T0, $\mathrm{p}=0.0009$ ). Mann-Whitney G1-G2 p=0.6041; Mann-Whitney G1-G4 p=0.0026; Mann-Whitney G2-G4 p=0.0006.

Table 2. Mean bleeding index (\%) of 45 patients: 15 from Group 1 (RA+PD+TR+), 15 from Group 2 (RA+PD+TR-) and 15 from Group 4 (RA-PD+TR+).

\begin{tabular}{lccccccc}
\hline Patients & $\begin{array}{c}\mathrm{T} 0 \\
\text { Initial }\end{array}$ & $\begin{array}{c}\mathrm{T} 1 \\
3 \text { months }\end{array}$ & $\begin{array}{c}\mathrm{T} 2 \\
6 \text { months }\end{array}$ & Friedman & $\begin{array}{c}\text { T1-T0 } \\
\text { Wilcoxon }\end{array}$ & $\begin{array}{c}\text { T2-T1 } \\
\text { Wilcoxon }\end{array}$ & $\begin{array}{c}\text { T2-T0 } \\
\text { Wilcoxon }\end{array}$ \\
\hline Group 1 & $49.47(35.19)$ & $25.91(28.93)$ & $29.78(28.93)$ & $\mathrm{p}<0.0726$ & $\mathrm{p}=0.0131$ & $\mathrm{p}=0.7537$ & $\mathrm{p}=0.0110$ \\
Group 2 & $34.25(18.53)$ & $34.25(18.53)$ & $34.25(18.53)$ & - & - & - & - \\
Group 4 & $21.19(7.92)$ & $12.06(6.21)$ & $7.62(7.15)$ & $\mathrm{p}<0.0000$ & $\mathrm{p}=0.0007$ & $\mathrm{p}=0.0007$ & $\mathrm{p}=0.0076$ \\
\hline
\end{tabular}

Mean (standard deviation). Kruskal-Wallis ANOVA (testing overall difference among the three groups in the baseline $-\mathrm{T} 0, \mathrm{p}=0.0467$ ). Mann-Whitney G1-G2 p=0.4937; Mann-Whitney G1-G4 p=0.0852; Mann-Whitney G2-G4 p=0.0114.

Table 3. Mean plaque index (\%) of 45 patients: 15 from Group 1 (RA+PD+TR+), 15 from Group 2 (RA+PD+TR-) and 15 from Group 4 (RA-PD+TR+).

\begin{tabular}{lccccccc}
\hline Patients & $\begin{array}{c}\mathrm{T} 0 \\
\text { Initial }\end{array}$ & $\begin{array}{c}\mathrm{T} 1 \\
\text { 3 months }\end{array}$ & $\begin{array}{c}\mathrm{T} 2 \\
6 \text { months }\end{array}$ & Friedman & $\begin{array}{c}\text { T1-T0 } \\
\text { Wilcoxon }\end{array}$ & $\begin{array}{c}\text { T2-T1 } \\
\text { Wilcoxon }\end{array}$ & $\begin{array}{c}\text { T2-T0 } \\
\text { Wilcoxon }\end{array}$ \\
\hline Group 1 & $83.98(25.64)$ & $44.24(32.28)$ & $42.90(32.17)$ & $\mathrm{p}<0.0016$ & $\mathrm{p}=0.0047$ & $\mathrm{p}=0.6379$ & $\mathrm{p}=0.0022$ \\
Group 2 & $66.08(18.94)$ & $66.08(18.94)$ & $66.08(18.94)$ & - & - & - & - \\
Group 4 & $51.35(8.77)$ & $35.29(16.61)$ & $17.92(12.99)$ & $\mathrm{p}<0.0000$ & $\mathrm{p}=0.0064$ & $\mathrm{p}=0.0007$ & $\mathrm{p}=0.0038$ \\
\hline
\end{tabular}

Mean (standard deviation). Kruskal-Wallis ANOVA (testing overall difference among the three groups in the baseline $-\mathrm{T} 0$, $\mathrm{p}=0.0017$ ). Mann-Whitney G1-G2 p=0.0400; Mann- Whitney G1-G4 p=0.0015; Mann-Whitney G2-G4 p=0.0294. 
the $0-3$ months with reductions in the AAG in the 3-6 months examinations $(\mathrm{r}=0.58 ; \mathrm{p}<0.0114)$.
In the groups with RA and PD the correlations were not found.

Table 4. Mean reduction of PPD (mm), \% BOP, \% PL between groups.

\begin{tabular}{|c|c|c|c|c|c|c|c|}
\hline Variables & Groups & $\begin{array}{c}\mathrm{T} 1-\mathrm{T} 0 \\
\text { mean } \pm \mathrm{sd}\end{array}$ & $\begin{array}{c}\mathrm{T} 2-\mathrm{T} 1 \\
\text { mean } \pm \mathrm{sd}\end{array}$ & $\begin{array}{c}\mathrm{T} 2-\mathrm{T} 0 \\
\text { mean } \pm \mathrm{sd}\end{array}$ & $\begin{array}{c}\text { T1-T0 } \\
\text { Mann } \\
\text { Whitney }\end{array}$ & $\begin{array}{c}\text { T2-T1 } \\
\text { Mann } \\
\text { Whitney }\end{array}$ & $\begin{array}{c}\text { T2-T0 } \\
\text { Mann } \\
\text { Whitney }\end{array}$ \\
\hline & Group 1 & $-0.42 \pm 0.50$ & $-0.11 \pm 0.27$ & $-0.54 \pm 0.49$ & \multirow{2}{*}{$\mathrm{p}=0.6334$} & \multirow{2}{*}{$\mathrm{p}=0.2717$} & \multirow{2}{*}{$\mathrm{p}=0.8846$} \\
\hline PPD & Group4 & $-0.35 \pm 0.24$ & $-0.24 \pm 0.32$ & $-0.59 \pm 0.23$ & & & \\
\hline \multirow[b]{2}{*}{$\% \mathrm{BOP}$} & Group 1 & $-23.56 \pm 32.53$ & $+3.87 \pm 16.31$ & $-19.69 \pm 28.53$ & \multirow{2}{*}{$\mathrm{p}=0.9339$} & \multirow{2}{*}{$\mathrm{p}=0.1985$} & \multirow{2}{*}{$\mathrm{p}=0.8519$} \\
\hline & Group 4 & $-9.13 \pm 4.40$ & $-4.43 \pm 4.83$ & $-13.56 \pm 4.68$ & & & \\
\hline \multirow[b]{2}{*}{$\% \mathrm{PL}$} & Group 1 & $-39.74 \pm 36.54$ & $-1.34 \pm 37.97$ & $-41.08 \pm 33.79$ & \multirow{2}{*}{$\mathrm{p}=0.1198$} & \multirow{2}{*}{$\mathrm{p}=0.0152$} & \multirow{2}{*}{$\mathrm{p}=0.7875$} \\
\hline & Group 4 & $-16.06 \pm 15.36$ & $-17.37 \pm 18.30$ & $-33.43 \pm 11.83$ & & & \\
\hline
\end{tabular}

$\mathrm{PPD}=$ probing depth; $\mathrm{BOP}=$ bleeding on probing; $\mathrm{PL}=$ Plaque; Group 1= RA+PD+TR+; Group 4= RA-PD+TR+.

Table 5. Mean reduction of PPD (mm), \% BOP, \% PL between groups.

\begin{tabular}{|c|c|c|c|c|c|c|c|}
\hline Variables & Groups & $\begin{array}{c}\mathrm{T} 1-\mathrm{T} 0 \\
\text { mean } \pm \mathrm{sd}\end{array}$ & $\begin{array}{c}\mathrm{T} 2-\mathrm{T} 1 \\
\text { mean } \pm \mathrm{sd}\end{array}$ & $\begin{array}{c}\mathrm{T} 2-\mathrm{T} 0 \\
\text { mean } \pm \mathrm{sd}\end{array}$ & $\begin{array}{c}\text { T1-T0 } \\
\text { Mann } \\
\text { Whitney }\end{array}$ & $\begin{array}{c}\text { T2-T1 } \\
\text { Mann } \\
\text { Whitney }\end{array}$ & $\begin{array}{c}\text { T2-T0 } \\
\text { Mann } \\
\text { Whitney }\end{array}$ \\
\hline \multirow{2}{*}{ PPD } & Group 1 & $-0.42 \pm 0.50$ & $-0.11 \pm 0.27$ & $-0.54 \pm 0.49$ & \multirow{2}{*}{$\mathrm{p}=0.0055$} & \multirow{2}{*}{$\mathrm{p}=0.2211$} & \multirow{2}{*}{$\mathrm{p}=0.0066$} \\
\hline & Group 2 & $-0.001 \pm 0.05$ & $-0.01 \pm 0.05$ & $-0.01 \pm 0.06$ & & & \\
\hline \multirow{2}{*}{$\% \mathrm{BOP}$} & Group 1 & $-23.56 \pm 32.53$ & $+3.87 \pm 16.31$ & $-19.69 \pm 28.53$ & \multirow{2}{*}{$\mathrm{p}=0.1198$} & \multirow{2}{*}{$\mathrm{p}=0.5338$} & \multirow{2}{*}{$\mathrm{p}=0.0128$} \\
\hline & Group 2 & - & - & - & & & \\
\hline \multirow{2}{*}{$\% \mathrm{PL}$} & Group 1 & $-39.74 \pm 36.54$ & $-1.34 \pm 37.97$ & $-41.08 \pm 33.79$ & \multirow{2}{*}{$\mathrm{p}=0.0128$} & \multirow{2}{*}{$\mathrm{p}=0.5338$} & \multirow{2}{*}{$\mathrm{p}=0.0002$} \\
\hline & Group 2 & - & - & - & & & \\
\hline
\end{tabular}

$\mathrm{PPD}=$ probing depth; $\mathrm{BOP}=$ bleeding on probing; $\mathrm{PL}=$ Plaque; Group 1= RA+PD+TR+; Group 2= RA+PD+TR-.

Table 6. Mean AAG (mg \%) of 45 patients: 15 from Group 1 (RA+PD+TR+), 15 from Group 2 (RA+PD+TR-) and 15 from Group 4 (RA-PD+TR+).

\begin{tabular}{lccccccc}
\hline Patients & $\begin{array}{c}\text { T0 } \\
\text { Initial }\end{array}$ & $\begin{array}{c}\text { T1 } \\
\text { 3 months }\end{array}$ & $\begin{array}{c}\text { T2 } \\
6 \text { months }\end{array}$ & Friedman & $\begin{array}{c}\text { T1-T0 } \\
\text { Wilcoxon }\end{array}$ & $\begin{array}{c}\text { T2-T1 } \\
\text { Wilcoxon }\end{array}$ & $\begin{array}{c}\text { T2-T0 } \\
\text { Wilcoxon }\end{array}$ \\
\hline Group 1 & $91.57(24.93)$ & $92.11(27.54)$ & $88.67(23.95)$ & $\mathrm{p}<0.9355$ & - & - & - \\
Group 2 & $102.13(31.96)$ & $101.67(36.33)$ & $120.47(16.13)$ & $\mathrm{p}<0.0707$ & - & - & - \\
Group 4 & $97.67(10.87)$ & $86.20(10.56)$ & $78.27(12.39)$ & $\mathrm{p}<0.0001$ & $\mathrm{p}=0.0031$ & $\mathrm{p}=0.0012$ & $\mathrm{p}=0.0018$ \\
\hline
\end{tabular}

Mean (standard deviation). Kruskal-Wallis ANOVA (testing overall difference among the 3 Groups in the baseline -T0, $\mathrm{p}=0.2416$ ). Mann-Whitney G1-G2 p=0.1585; Mann-Whitney G1-G4 p=0.1409; Mann-Whitney G2-G4 p=0.7557. 


\section{DISCUSSION}

Chronic periodontal disease can be considered a potential focus of infection, which worsens the meta- bolic control of patients with diabetes and RA(11). The pathobiology of periodontal disease (PD) and rheumatoid arthritis (RA) is similar, both are inflammatory chronic diseases, with activation of complement, production of

Table 7. Mean CRP (mg \%) of 45 patients: 15 from Group 1 (RA+PD+TR+), 15 from Group 2 (RA+PD+TR-) and 15 from Group 4 (RA-PD+TR+).

\begin{tabular}{lccccccc}
\hline Patients & $\begin{array}{c}\text { T0 } \\
\text { Initial }\end{array}$ & $\begin{array}{c}\text { T1 } \\
\text { 3 months }\end{array}$ & $\begin{array}{c}\text { T2 } \\
6 \text { months }\end{array}$ & Friedman & $\begin{array}{c}\text { T1-T0 } \\
\text { Wilcoxon }\end{array}$ & $\begin{array}{c}\text { T2-T1 } \\
\text { Wilcoxon }\end{array}$ & $\begin{array}{c}\text { T2-T0 } \\
\text { Wilcoxon }\end{array}$ \\
\hline Group 1 & $0.90(1.06)$ & $0.86(1.11)$ & $0.63(0.54)$ & $\mathrm{p}<0.4203$ & - & - & - \\
Group 2 & $0.61(0.19)$ & $0.55(0.18)$ & $0.81(1.10)$ & $\mathrm{p}<0.9252$ & - & - & - \\
Group 4 & $2.07(1.26)$ & $1.23(0.77)$ & $0.63(0.31)$ & $\mathrm{p}<0.0000$ & $\mathrm{p}=0.0024$ & $\mathrm{p}=0.0022$ & $\mathrm{p}=0.0015$ \\
\hline
\end{tabular}

Mean (standard deviation). Kruskal-Wallis ANOVA (testing overall difference among the three groups in the baseline - T0, $\mathrm{p}=0.071$ ). Mann-Whitney G1-G2 p=0.2902; Mann- Whitney G1-G4 p=0.0136; Mann-Whitney G2-G4 p=0.0045.

Table 8. Mean ESR (mm/h) of 45 patients: 15 from Group 1 (RA+PD+TR+), 15 from Group 2 (RA+PD+TR-) and 15 from Group 4 (RA-PD+TR+).

\begin{tabular}{|c|c|c|c|c|c|c|c|}
\hline Patients & $\begin{array}{c}\text { T0 } \\
\text { Initial }\end{array}$ & $\begin{array}{c}\text { T1 } \\
3 \text { months }\end{array}$ & $\begin{array}{c}\text { T2 } \\
6 \text { months }\end{array}$ & Friedman & $\begin{array}{c}\text { T1-T0 } \\
\text { Wilcoxon }\end{array}$ & $\begin{array}{c}\text { T2-T1 } \\
\text { Wilcoxon }\end{array}$ & $\begin{array}{c}\text { T2-T0 } \\
\text { Wilcoxon }\end{array}$ \\
\hline Group 1 & $24.53(13.13)$ & $24.07(13.51)$ & $22.40(13.13)$ & $\mathrm{p}<0.6449$ & - & - & - \\
\hline Group 2 & $34.80(18.08)$ & $39.40(16.37)$ & $28.33(8.47)$ & $\mathrm{p}<0.0030$ & $\mathrm{p}=0.0535$ & $\mathrm{p}=0.3109$ & $\mathrm{p}=0.0007$ \\
\hline Group 4 & $37.47(7.36)$ & $34.67(9.59)$ & $27.67(9.60)$ & $\mathrm{p}<0.0000$ & $\mathrm{p}=0.0106$ & $\mathrm{p}=0.0007$ & $\mathrm{p}=0.0007$ \\
\hline
\end{tabular}

Mean (standard deviation). KrusKal-Wallis Anova (testing overall difference among the three groups in the baseline - T0, $\mathrm{p}=0.0158$ ). Mann-Whitney G1-G2 p=0.1647; Mann-Whitney G1-G4 p=0.0035; Mann-Whitney G2-G4 p=0.1776.

Table 9. Mean reduction of AAG (mg \%), CRP ( $\mathrm{mg} \%)$, ERS ( $\mathrm{mm} / \mathrm{h})$ between groups.

\begin{tabular}{|c|c|c|c|c|c|c|c|}
\hline Variables & Groups & $\begin{array}{c}\mathrm{T} 1-\mathrm{T} 0 \\
\text { mean } \pm \text { sd }\end{array}$ & $\begin{array}{c}\mathrm{T} 2-\mathrm{T} 1 \\
\text { mean } \pm \mathrm{sd}\end{array}$ & $\begin{array}{c}\mathrm{T} 2-\mathrm{T} 0 \\
\text { mean } \pm \mathrm{sd}\end{array}$ & $\begin{array}{c}\text { T1-T0 } \\
\text { Mann } \\
\text { Whitney }\end{array}$ & $\begin{array}{c}\text { T2-T1 } \\
\text { Mann } \\
\text { Whitney }\end{array}$ & $\begin{array}{c}\text { T2-T0 } \\
\text { Mann } \\
\text { Whitney }\end{array}$ \\
\hline \multirow{2}{*}{ AAG } & Group 1 & $+0.54 \pm 27.01$ & $-3.44 \pm 18.09$ & $-2.90 \pm 20.10$ & \multirow{2}{*}{$\mathrm{p}=0.0381$} & \multirow{2}{*}{$\mathrm{p}=0.2902$} & \multirow{2}{*}{$\mathrm{p}=0.0144$} \\
\hline & Group 4 & $-11.47 \pm 9.65$ & $-7.93 \pm 5.80$ & $-19.40 \pm 12.56$ & & & \\
\hline \multirow{2}{*}{ CRP } & Group 1 & $-0.04 \pm 1.36$ & $-0.23 \pm 0.89$ & $-0.27 \pm 0.92$ & \multirow{2}{*}{$\mathrm{p}=0.1095$} & \multirow{2}{*}{$\mathrm{p}=0.0023$} & \multirow{2}{*}{$\mathrm{p}=0.0015$} \\
\hline & Group 4 & $-0.84 \pm 1.12$ & $-0.59 \pm 0.57$ & $-1.43 \pm 1.19$ & & & \\
\hline \multirow{2}{*}{ ESR } & Group 1 & $-0.47 \pm 9.44$ & $-1.67 \pm 6.04$ & $-2.13 \pm 3.46$ & \multirow{2}{*}{$\mathrm{p}=0.6182$} & \multirow{2}{*}{$\mathrm{p}=0.0016$} & \multirow{2}{*}{$\mathrm{p}=0.0019$} \\
\hline & Group 4 & $-2.80 \pm 5.35$ & $-7.00 \pm 4.50$ & $-9.80 \pm 5.76$ & & & \\
\hline
\end{tabular}

$\mathrm{AAG}=$ alpha- 1 acid glycoprotein; $\mathrm{CRP}=\mathrm{C}$-reactive protein; $\mathrm{ESR}=$ The erythrocyte sedimentation rate. $\mathrm{Group} 1=\mathrm{RA}+\mathrm{PD}+\mathrm{TR}+$; Group 4= RA-PD+TR+. 
cytokines and release of other inflammatory cell products $(12,13)$. However, the relationship between the 2 diseases is controversial, due to difficulties in analyzing the association between disease activities of RA and PD in randomized controlled studies, as described by Ribeiro et al. (14) and Miranda el al. (15). Therefore, further studies are needed to study the relationship between the 2 diseases $(4,16)$.

In the present study, the improvement in Group 1 $(\mathrm{RA}+\mathrm{PD}+\mathrm{TR}+$ ) after non-surgical periodontal treatment (NSPT) was statistically significant in the control of the periodontal disease, but the systemic values, although reduced, were not statistically significant. (Tables 1-3,6-8).

When the Groups 1 (RA+PD+TR+), 2 (RA+DP+TR-) and 4 (RA-DP+TR+) were compared at baseline, they were statistically different. This fact does not compromise the study, because in the comparison between groups, the reduction values were used instead of the means.

Periodontal status improvement affecting the laboratory results (acute-phase proteins) was easier to be noted in Group 4, meaning that RA is a multifactorial disease, which makes the control of the other inflammatory processes in the organism difficult (4). In Group 4 there was a correlation between the reduction in \% of BOP, PPD, $\%$ of PL and reductions in AAG.

Tables 4 and 9 compares Groups 1 and 4, showing reductions in periodontal and systemic values after NSPT. There are increased reductions for Group 4 in $\%$ of PL (T2-T1), AAG (T1-T0, T2-T0), CRP (T2-T1, T2-T0) and ESR (T2-T1, T2-T0). This result could be due to the initial differences between Groups, observing the initial mean values and their standard deviations. The $\%$ of PL was lower in Group 4, in part, because of the patients' compliance, since patients of Group 1 were less motivated, considering emotional factors and physical limitations caused by the symptoms of RA

Comparing Groups with RA, 1 and 2, large reductions were noted in the Groups treated in all the parameters (periodontal, systemic and specific RA tests). This result suggests the necessity of patients with RA to receive periodontal treatment, even though the correlation was not totally clear. It could be explained by the difficulties to work with multifactorial systemic

Table 10. Mean reduction of AAG (mg \%), CRP (mg \%), ERS (mm/h), PPD (mm), \% BOP, \% PL between groups.

\begin{tabular}{|c|c|c|c|c|c|c|c|}
\hline Variables & Groups & $\begin{array}{c}\text { Initial } \\
\text { mean } \pm \text { sd }\end{array}$ & $\begin{array}{l}3 \text { months } \\
\text { mean } \pm \text { sd }\end{array}$ & $\begin{array}{l}6 \text { months } \\
\text { mean } \pm \text { sd }\end{array}$ & $\begin{array}{c}\text { Mann- } \\
\text { Whitney } \\
\text { initial }\end{array}$ & $\begin{array}{l}\text { Mann- } \\
\text { Whitney } \\
3 \text { months }\end{array}$ & $\begin{array}{l}\text { Mann- } \\
\text { Whitney } \\
6 \text { months }\end{array}$ \\
\hline \multirow{2}{*}{ AAG } & Group 5 & & $57.13 \pm 13.39$ & & \multirow{2}{*}{$\mathrm{p}=0.0000$} & \multirow{2}{*}{$\mathrm{p}=0.0000$} & \multirow{2}{*}{$\mathrm{p}=0.0004$} \\
\hline & Group 4 & $97.67 \pm 10.89$ & $86.20 \pm 10.56$ & $78.27 \pm 12.39$ & & & \\
\hline \multirow{2}{*}{ CRP } & Group 5 & & $0.61 \pm 0.28$ & & \multirow{2}{*}{$\mathrm{p}=0.0020$} & \multirow{2}{*}{$\mathrm{p}=0.0171$} & \multirow{2}{*}{$\mathrm{p}=0.9670$} \\
\hline & Group 4 & $2.07 \pm 1.26$ & $1.23 \pm 0.77$ & $0.63 \pm 0.31$ & & & \\
\hline \multirow{2}{*}{ ESR } & Group 5 & & $20.60 \pm 8.70$ & & \multirow{2}{*}{$\mathrm{p}=0.0001$} & \multirow{2}{*}{$\mathrm{p}=0.0007$} & \multirow{2}{*}{$\mathrm{p}=0.0591$} \\
\hline & Group 4 & $37.47 \pm 7.36$ & $34.67 \pm 9.59$ & $27.67 \pm 9.60$ & & & \\
\hline \multirow{2}{*}{ PPD } & Group 5 & & $2.29 \pm 0.10$ & & \multirow{2}{*}{$\mathrm{p}=0.6936$} & \multirow{2}{*}{$\mathrm{p}=0.4186$} & \multirow{2}{*}{$\mathrm{p}=0.0005$} \\
\hline & Group 4 & $2.46 \pm 0.47$ & $2.10 \pm 0.51$ & $1.87 \pm 0.39$ & & & \\
\hline \multirow[t]{2}{*}{$\%$ BOP } & Group 5 & & $1.71 \pm 0.98$ & & \multirow{2}{*}{$\mathrm{p}=0.0000$} & \multirow{2}{*}{$\mathrm{p}=0.0000$} & \multirow{2}{*}{$\mathrm{p}=0.0017$} \\
\hline & Group 4 & $21.18 \pm 7.92$ & $12.06 \pm 6.21$ & $7.63 \pm 7.15$ & & & \\
\hline \multirow{2}{*}{$\% \mathrm{PL}$} & Group 5 & & $43.73 \pm 6.11$ & & \multirow{2}{*}{$\mathrm{p}=0.0084$} & \multirow{2}{*}{$\mathrm{p}=0.0712$} & \multirow{2}{*}{$\mathrm{p}=0.0001$} \\
\hline & Group 4 & $51.35 \pm 8.77$ & $35.29 \pm 16.61$ & $17.92 \pm 12.99$ & & & \\
\hline
\end{tabular}

$\mathrm{AAG}=$ alpha-1 acid glycoprotein; $\mathrm{CRP}=\mathrm{C}$-reactive protein; $\mathrm{ESR}=$ The erythrocyte sedimentation rate $\mathrm{PPD}=$ probing depth; $\mathrm{BOP}=$ bleeding on probing; PL = Plaque. Group 4 = RA-PD+TR+; Group 5 =RA-PD-. 
diseases and the difficulties of getting a larger numbers of patients for each Group ( $\mathrm{n}=15)$.

Comparing Groups 4 (RA-DP+TR+) and 5 (RA-PD-), PPD was not statistically significant only at baseline. After 3 months PPD and \% of PL and after 6 months CRP and ESR were not statistically different. This shows the reduction of acute-phase proteins by the periodontal treatment and that after 6 months the test Group became similar to the control Group, demonstrating a healthy profile.

This same observation was difficult to be detected when comparing Groups 1 (RA+PD+TR+) and 5 (RAPD-) because the values were statistically significant: AAG and $\%$ of BOP were maintained during the evaluation periods, despite the reductions in the test group. \% of PL was not statistically different after 3 months and PPD after 6 months. The improvement of PPD and \% of PL was evident, but not for the systemic parameters. This could be explained by the continuous use of systemic medications (SAIDS and NSAIDS drugs) by RA patients that results in control of the acute phase proteins and
RA, and since RA is a multifactorial systemic disease, the clinical improvements, after periodontal treatment, are more difficult to be detected.

Considering Group 1, the periodontal improvement did not correlate to the laboratory values. This could be due to the small size of the sample, with non-normal distributions, being evident the necessity of a descriptive analysis of the data.

So, even if the correlations were not so clear, considering the reduction values and the dispersion points in the correlation graphs, it was possible to see that the laboratory results and specific tests for RA improved or were controlled by the periodontal treatment.

Analyzing the periodontal status, the patients from Group $1(\mathrm{RA}+\mathrm{PD}+\mathrm{TR}+)$ were similar to healthy patients, although their acute phase proteins presented higher values. The chronic used drugs (SAIDS and NSAIDS) by the RA patients to mitigate symptoms of the disease could affect the progression of bone loss, but not the clinical periodontal improvement (16-18). Thus, the difficulty to show a correlation between RA and PD

Table 11. Mean reduction of AAG (mg \%), CRP (mg \%), ERS (mm/h), PPD (mm), \% BOP, \% PL between groups.

\begin{tabular}{|c|c|c|c|c|c|c|c|}
\hline Variables & Groups & $\begin{array}{c}\text { Initial } \\
\text { mean } \pm \text { sd }\end{array}$ & $\begin{array}{l}3 \text { months } \\
\text { mean } \pm \text { sd }\end{array}$ & $\begin{array}{l}6 \text { months } \\
\text { mean } \pm \text { sd }\end{array}$ & $\begin{array}{c}\text { Mann- } \\
\text { Whitney } \\
\text { initial }\end{array}$ & $\begin{array}{l}\text { Mann- } \\
\text { Whitney } \\
3 \text { months }\end{array}$ & $\begin{array}{c}\text { Mann- } \\
\text { Whitney } \\
6 \text { months }\end{array}$ \\
\hline \multirow{2}{*}{$\mathrm{AAG}$} & Group 5 & & & & \multirow{2}{*}{$\mathrm{p}=0.0002$} & \multirow{2}{*}{$\mathrm{p}=0.0001$} & \multirow{2}{*}{$\mathrm{p}=0.0001$} \\
\hline & Group 1 & $91.57 \pm 24.93$ & $92.11 \pm 27.54$ & $88.67 \pm 23.95$ & & & \\
\hline \multirow{2}{*}{ CRP } & Group 5 & & $0.61 \pm 0.28$ & & \multirow{2}{*}{$\mathrm{p}=0.5897$} & \multirow{2}{*}{$\mathrm{p}=0.3297$} & \multirow{2}{*}{$p=0.6041$} \\
\hline & Group 1 & $0.90 \pm 1.06$ & $0.86 \pm 1.11$ & $0.63 \pm 0.54$ & & & \\
\hline \multirow[t]{2}{*}{ ESR } & Group 5 & & $20.60 \pm 8.70$ & & \multirow{2}{*}{$\mathrm{p}=0.2998$} & \multirow{2}{*}{$\mathrm{p}=0.6187$} & \multirow{2}{*}{$\mathrm{p}=0.8682$} \\
\hline & Group 1 & $24.53 \pm 13.13$ & $24.07 \pm 13.51$ & $22.40 \pm 13.13$ & & & \\
\hline \multirow{2}{*}{ PPD } & Group 5 & & $2.29 \pm 0.10$ & & \multirow{2}{*}{$\mathrm{p}=0.0001$} & \multirow{2}{*}{$\mathrm{p}=0.0095$} & \multirow{2}{*}{$\mathrm{p}=0.0779$} \\
\hline & Group 1 & $3.24 \pm 0.88$ & $2.82 \pm 0.77$ & $2.71 \pm 0.76$ & & & \\
\hline \multirow[t]{2}{*}{$\%$ BOP } & Group 5 & & $1.71 \pm 0.98$ & & \multirow{2}{*}{$\mathrm{p}=0.0000$} & \multirow{2}{*}{$\mathrm{p}=0.0000$} & \multirow{2}{*}{$\mathrm{p}=0.0000$} \\
\hline & Group 1 & $49.47 \pm 35.19$ & $25.91 \pm 28.93$ & $29.78 \pm 28.93$ & & & \\
\hline \multirow{2}{*}{$\% \mathrm{PL}$} & Group 5 & & $43.73 \pm 6.11$ & & \multirow{2}{*}{$\mathrm{p}=0.0004$} & \multirow{2}{*}{$\mathrm{p}=0.0852$} & \multirow{2}{*}{$\mathrm{p}=0.1409$} \\
\hline & Group 1 & $83.98 \pm 25.64$ & $44.24 \pm 32.28$ & $42.90 \pm 32.17$ & & & \\
\hline
\end{tabular}

$\mathrm{AAG}=$ alpha- 1 acid glycoprotein; $\mathrm{CRP}=\mathrm{C}$-reactive protein; $\mathrm{ESR}=$ The erythrocyte sedimentation rate.

$\mathrm{PPD}=$ probing depth; $\mathrm{BOP}=$ bleeding on probing; $\mathrm{PL}=$ Plaque.

Group 1= RA+PD+TR+; Group5=RA-PD-. 
could be associated with the chronic use of drugs. The fact that many of the clinical studies available comparing the periodontal status to RA are transversal studies increases the difficulties in evaluating important points such as tooth loss $(8,19)$.

In Groups $1(\mathrm{RA}+\mathrm{PD}+\mathrm{TR}+)$ and $3(\mathrm{RA}+\mathrm{TP})$ there was a statistical difference at baseline and the 3 months evaluation for the DAS28, but not for the 6 months evaluation, favoring Group 1 . This shows that RA patients do not need to have their teeth extracted in order to control the disease, point which was raised also by PAGE (20).

The control of the inflammatory activity improved the periodontal status in systemically healthy patients. Although there was an improvement in the periodontal status of RA patients, the inflammatory activity was not significantly reduced. This can be explained by the fact that RA patients have other sites that contribute to the inflammatory panorama besides the oral cavity.

In conclusion, although the relation between $\mathrm{RA}$ and PD, is not clear, it is possible to indicate the importance of the periodontal treatment in the control of inflammation, in order to avoid tooth extractions and to guarantee benefits for the patients with RA.

\section{RESUMO}

Este estudo avaliou a influência da doença periodontal (DP) na artrite reumatóide (AR). 75 pacientes, 35-60 anos, divididos em 5 Grupos, considerando a situação sistêmica e periodontal foram analisados. Um Grupo usava prótese total (PT). Os parâmetros

Table 12. Mean reduction of AAG (mg \%), CRP (mg \%), ERS (mm/h), PPD (mm), \% BOP, \% PL between groups.

\begin{tabular}{|c|c|c|c|c|c|c|c|}
\hline Variables & Groups & $\begin{array}{c}\text { Initial } \\
\text { mean } \pm \text { sd }\end{array}$ & $\begin{array}{l}3 \text { months } \\
\text { mean } \pm \text { sd }\end{array}$ & $\begin{array}{l}6 \text { months } \\
\text { mean } \pm \text { sd }\end{array}$ & $\begin{array}{l}\text { Mann- } \\
\text { Whitney } \\
\text { initial }\end{array}$ & $\begin{array}{c}\text { Mann- } \\
\text { Whitney } \\
3 \text { months }\end{array}$ & $\begin{array}{l}\text { Mann- } \\
\text { Whitney } \\
6 \text { months }\end{array}$ \\
\hline \multirow{2}{*}{ AAG } & Group 1 & $91.57 \pm 24.93$ & $92.11 \pm 27.54$ & $88.67 \pm 23.95$ & \multirow{2}{*}{$\mathrm{p}=0.9010$} & \multirow{2}{*}{$\mathrm{p}=0.9504$} & \multirow{2}{*}{$\mathrm{p}=0.6187$} \\
\hline & Group 3 & & $90.72 \pm 21.96$ & & & & \\
\hline \multirow{2}{*}{ CRP } & Group 1 & $0.90 \pm 1.06$ & $0.86 \pm 1.11$ & $0.63 \pm 0.54$ & \multirow{2}{*}{$\mathrm{p}=0.9174$} & \multirow{2}{*}{$\mathrm{p}=0.9339$} & \multirow{2}{*}{$\mathrm{p}=0.9010$} \\
\hline & Group 3 & & $0.79 \pm 0.83$ & & & & \\
\hline \multirow[t]{2}{*}{ ESR } & Group 1 & $24.53 \pm 13.13$ & $24.07 \pm 13.51$ & $22.40 \pm 13.13$ & \multirow{2}{*}{$\mathrm{p}=07875$} & \multirow{2}{*}{$\mathrm{p}=04679$} & \multirow{2}{*}{$\mathrm{p}=0.3837$} \\
\hline & Group 3 & & $27.20 \pm 15.20$ & & & & \\
\hline \multirow[t]{2}{*}{ HAQ } & Group 1 & $1.22 \pm 0.81$ & $1.16 \pm 0.71$ & $0.91 \pm 0.54$ & \multirow{2}{*}{$\mathrm{p}=0.2290$} & \multirow{2}{*}{$\mathrm{p}=0.1844$} & \multirow{2}{*}{$\mathrm{p}=0.4807$} \\
\hline & Group 3 & & $0.83 \pm 0.64$ & & & & \\
\hline \multirow{2}{*}{ DAS28 } & Group 1 & $3.34 \pm 1.14$ & $2.69 \pm 0.88$ & $2.25 \pm 0.66$ & \multirow{2}{*}{$\mathrm{p}=0.0062$} & \multirow{2}{*}{$\mathrm{p}=0.0381$} & \multirow{2}{*}{$\mathrm{p}=0.5755$} \\
\hline & Group 3 & & $2.14 \pm 0.76$ & & & & \\
\hline \multirow{2}{*}{ SFA } & Group 1 & $52.67 \pm 31.05$ & $62.33 \pm 24.70$ & $64.33 \pm 25.13$ & \multirow{2}{*}{$\mathrm{p}=0.2540$} & \multirow{2}{*}{$\mathrm{p}=0.6482$} & \multirow{2}{*}{$\mathrm{p}=1$} \\
\hline & Group 3 & & $66.00 \pm 15.61$ & & & & \\
\hline \multirow{2}{*}{ SFB } & Group 1 & $54.67 \pm 41.25$ & $68.67 \pm 28.88$ & $72.33 \pm 25.97$ & \multirow{2}{*}{$\mathrm{p}=0.8035$} & \multirow{2}{*}{$\mathrm{p}=0.4807$} & \multirow{2}{*}{$\mathrm{p}=0.1985$} \\
\hline & Group 3 & & $61.33 \pm 15.75$ & & & & \\
\hline \multirow{2}{*}{ SFC } & Group 1 & $50.13 \pm 30.52$ & $64.40 \pm 25.69$ & $66.23 \pm 24.56$ & \multirow{2}{*}{$\mathrm{p}=0.1914$} & \multirow{2}{*}{$=0.8682$} & \multirow{2}{*}{$\mathrm{p}=0.8357$} \\
\hline & Group 3 & & $64.80 \pm 18.85$ & & & & \\
\hline
\end{tabular}

$\mathrm{AAG}=$ alpha- 1 acid glycoprotein; $\mathrm{CRP}=\mathrm{C}$-reactive protein; $\mathrm{ESR}=$ The erythrocyte sedimentation rate.

$\mathrm{HAQ}=$ The Stanford Health Assessment; DAS28= Disease Activity Score; SF36= Short-Form Health Survey.

Group 1= RA+PD+TR+; Group 3=RA+TP. 
clínicos e laboratoriais analisados: inicial, 3 e 6 meses receberam tratamento estatístico. As correlações entre as reduções da profundidade de sondagem, placa microbiana e sangramento à sondagem com as de Alfa 1, PCR, VHS e exames de qualidade de vida (HAQ, DAS28, SF36) foram também analisadas. Comparando o Grupo $1(\mathrm{AR}+\mathrm{DP}+\mathrm{TR}+)$ e Grupo $2(\mathrm{AR}+\mathrm{DP}+\mathrm{TR}-)$, aqueles que receberam tratamento periodontal não-cirúrgico (TPNC) tiveram reduções estatisticamente significativas em relação a PS aos 3 meses $(p=0,0055)$ e 6 meses $(p=0,0066)$; as reduções da \% SS foram estatisticamente significantes aos 6 meses $(\mathrm{p}=0,0128)$ e da \% PM aos $3(p=0,0128)$ e 6 meses $(p=0,0002)$. O TPNC não apresentou correlação com a melhora dos exames laboratoriais, nem com os exames de qualidade de vida. Comparando o Grupo 1 com o Grupo 3 (AR+PT) houve diferença estatisticamente significante no DAS28 inicial e 3 meses. Não foi possível verificar uma correlação direta do TPNC na atividade da doença sistêmica, mas pode-se constatar a sua importância, evitando a extração múltipla dos dentes.

\section{ACKNOWLEDGEMENTS}

The authors applied the SDC (sequence-determined-credit) approach for the sequence of authors.

\section{REFERENCES}

1. AAP - American Academy of Periodontology. Position paper Periodontal disease as a risk factor for other diseases. J Periodontol 1998;69:841-850.

2. Beck JD, Garcia RG, Heiss G, Vokonas PS, Offenbacher S. Periodontal disease and cardiovascular disease. J Periodontol 1996;67:1123-1137.

3. Ebersole JL. Systemic humoral immune response in periodontal disease. Crit Rev Oral Biol Med 1990;1:283-331.

4. Bartold PM, Marshall RI, Haynes DR. Periodontitis and rheumatoid arthritis: a review. J Periodontol 2005;76:2066-2074.

5. Sjostrom L, Laurell L, Hugoson A, Hakansson J. Periodontol conditions in adults with rheumatoid arthritis. Comm Dent Oral Epidemiol 1989;17:234-236.

6. Yavuzyilmaz E, Yamalik N, Calguner M, Ersov F, Baykara M, Yeniay I. Clinical and immunological characteristics of patients with rheumatoid arthritis and periodontal disease. J Nihon Univ Sch Dent 1992;34:89-95.
7. Kasser UR, Gleissner C, Dehne F, Michel A, WillershausenZönncher B, Bolten WW. Risk for periodontal disease in patients with longstanding rheumatoid arthritis. Arthritis and Rheum 1997;40:2248-2251.

8. Mercado FB, Marshall RI, Klestov AC, Bartold PM. Relationship between rheumatoid arthritis and periodontitis. J Periodontol 2001;72:779-787.

9. Arnett F, Edworthy S, Bloch D. The American rheumatism association 1987 revised criteria for the classification of rheumadoid arthritis. Arthritis \& Rheum 1988;31:315-324.

10. Machtei EE, Christersson LA, Grossi SG, Dunford R, Zambon JJ, Genco RJ. Clinical criteria for the definition of "Established Periodontitis". J Periodontol 1992;63:207-215.

11. Slots J. Casual or causal relationship between periodontal infection and non-oral disease? J Dent Res 1998;77:1764-1765.

12. Petty RE, Southwood TR, Manners P, Baum J, Glass DN, Goldenberg J, et al.. International league of associations for rheumatology classification of juvenile idiopathic arthritis: second revision, Edmonton, 2001. J Rheumatol 2004;31:390-392.

13. Smolik I, Robinson D, El-Gabalawy HS. Periodontitis and rheumatoid arthritis: epidemiologic, clinical, and immunologic associations.Compend Contin Educ Dent 2009;30:188-190.

14. Ribeiro J, Leão A, Novaes AB. Periodontal infection as a possible severity factor for rheumatoid arthritis. J Clin Periodontol 2005;32:412-416.

15. Miranda LA, Braga F, Fischer RG, Sztajnbok FR, Figueredo CMS, Gustafsson A. Changes in periodontal and rheumatological conditions after 2 years in patients with juvenile idiopathic arthritis. J Periodontol 2006;77:1695-1700.

16. Braga FSFF, Miranda LA, Miceli VC, Áreas A, Figueredo CMS, Fischer RG, et al.. Chronic arthritis and periodontitis. Rev Bras Reumatol 2007;47:276-280.

17. Feldman RS. Non-steroidal anti-inflammatory drugs in the reduction of human alveolar bone loss. J Clin Periodontol 1983;10:131136.

16. Heasman PA, Seymor RA. An association between long-term non-steroidal anti-inflammatory drug therapy and the severity of periodontal disease. J Clin Periodontol 1990;17:654-658.

19. Poulsen AH, Westergaard J, Stoltze K, Skjodt H, Samsoe BD, Locht $\mathrm{H}$, et al.. Periodontal and hematological characteristics associated with aggressive periodontitis, juvenile idiopatic arthritis, and rheumatoid arthritis. J Periodontol 2006;77:280-288.

20. Page RC. The pathobiology of periodontal diseases may affect systemics diseases: inversion of a paradigm. Ann Periodontol 1998;3:108-120. 